\title{
Patient advocacy and the future of systemic therapies in liver cancer: an interview with Ghassan Abou-Alfa
}

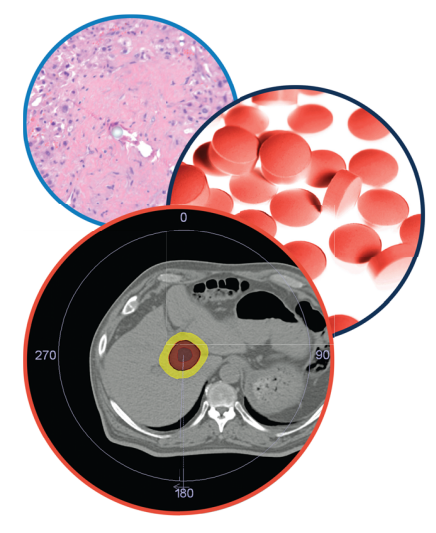

Hepatic Oncology

Ghassan Abou-Alfa*

*Author for correspondence: abou-alg@MSKCC.ORG

Discussing the importance of patient advocacy and the advancement of systemic therapies for the treatment of liver cancer, we catch up with Hepatic Oncology's latest editorial board member Ghassan AbouAlfa.

First draft submitted: 16 December 2019; Accepted for publication: 10 March 2020; Published online: 31 March 2020

Keywords: advocacy • cabozantinib • hepatocellular carcinoma • systemic therapies $\bullet$ tyrosine kinase inhibitors

Please introduce yourself \& give an overview of your career to date

My name is Ghassan Abou-Alfa, I'm an attending physician at Memorial Sloan Kettering Cancer Center (NY, USA). I work and help with the development of novel therapies for the treatment of liver cancer, cholangiocarcinoma, gallbladder cancer and fibrolamellar carcinoma.

What was the motivation to write your book "100 Questions \& Answers About Liver Cancer"? Many of our Memorial Sloan Kettering Hepatobiliary team members felt that there was no resource for patients to use and benefit from in order to learn more about the disease. Therefore, it was appropriate and valuable to prepare one. The publishers recognized the opportunity, so Ronald DeMatteo, now at the University of Pennsylvania (PA, USA) and I collaborated and wrote the book, which is now in its the fourth edition!

What do you think needs to be done to increase advocacy among liver cancer patients?

Our job is to respond to the patients' need for a cure. Thankfully there are curative options for patients including liver transplants, surgery and radio frequency ablation. Sadly, these options are only available to a small number of patients, approximately $15 \%$, and in most patient's liver cancer will recur, or present with locally advanced disease or metastatic disease. Even patients with locally advanced disease may ultimately develop metastatic disease, and thus require systemic therapies. In the last decade and before, there were no standards, there was little choice and we were dependent upon default options based on previous experiences with chemotherapy from other cancers. We were honored to have led from Memorial Sloan Kettering the first effort evaluating sorafenib, the first biological therapy for the treatment of liver cancer. From that point on, things progressed at a slow pace until 4 years ago when new advances in tyrosine kinase inhibitors (TKIs) and checkpoint inhibitors evolved rapidly and vastly increased the treatment options available for liver cancer.

\section{Do you think that as more TKI \& checkpoint inhibitor therapies are developed that patients will engage with them?}

TKIs are effective, and the newer ones are better tolerated. The new advances helped efface the perceived initial limitations and now TKIs have a clear and well-defined role in the treatment of the liver cancer.

\section{What has been the highlight of your career so far?}

I am honored to have witnessed a career that runs in parallel with the advancement of therapies for liver cancer. As previously mentioned, we proudly led the first Phase II study of sorafenib from Memorial Sloan Kettering. We were part of several collaborative efforts examining different therapeutic options, that for example helped add 
cabozantinib onto the platform of systemic therapies for liver cancer. I was also involved in evolving the use of combination checkpoint inhibitor therapies. The achieved advances are collectively great, but we shouldn't forget the patient need for a cure, thus our efforts should focus on better understanding and definition of the appropriate applications of CAR-T therapy for liver cancer.

\section{Can you give an overview of the results of the CELESTIAL trial?}

The CELESTIAL trial examined the value of cabozantinib, a TKI with a focus on VEGF receptors 1, 2, and 3, MET, and AXL. The trial results demonstrated that versus a placebo in both second- and third-line therapies an improvement of survival, as reported in the New England Journal of Medicine.

\section{Do you foresee US FDA approval of more TKIs for the treatment of hepatocellular carcinoma in the near future?}

The FDA have already approved five TKIs, which are sorafenib and lenvatinib as first line treatments. In the second line there is regorafenib with conditional progression on prior-sorafenib, ramucirumab requiring a high AFP level and cabozantinib which is approved as a second- and third-line therapy. We hope that these treatment options will soon be available worldwide.

\section{Will cabozantinib be demonstrated to be effective in combination therapies?}

Scientifically, a combination of cabozantinib and checkpoint inhibitors present an intriguing scientific component. The value of such a treatment would be incredibly important to examine, contributing to several other studies looking at combination therapies. The IMbrave 150 study evaluating atezolizumab plus bevacizumab demonstrated positive results and was presented at ESMO Asia Congress 2019 (Singapore, 22-24 November 2019). There is also lenvatinib plus pembrolizumab, and lastly a different approach investigating the addition of two checkpoint inhibitors durvalumab and tremelimumab.

The combinations including checkpoint inhibitor are not necessarily all the same. There are so far three types of combinations with the first two being closely related. The antiangiogenic inhibitors combined with an anti PD-L1, for example atezolizumab plus bevacizumab. Second there are TKIs combined with an anti PD-L1, for example lenvatinib plus pembrolizumab or cabozantinib plus nivolumab. And third there are the combination of different checkpoint inhibitors like durvalumab and tremelimumab. We may end up perceiving these three categories as different therapeutic approaches. We are still waiting on the outcomes of several trials to provide the details of how treatment options will evolve accordingly.

\section{In your opinion, what are the current challenges in developing systemic therapies for the treatment of hepatocellular carcinoma?}

The interest in the disease is thankfully evolving. Several current options are only available in North America, Western Europe and Japan. One would like to envision that these choices become readily available for patients worldwide. We should not forget the high incidence of liver cancer in Asia, as well as Africa.

\section{Why do you think sponsor support has increased for these therapies?}

The main reason is the high-quality science. The credit is due to all those involved in bringing a better understanding of the disease and therapeutic approaches leading to effective treatments.

Disclaimer

The opinions expressed in this interview are those of the interviewee and do not necessarily reflect the views of Future Medicine Ltd.

Financial \& competing interests disclosure

Reported potential conflicts of interest include research grants from ActaBiologica, Agios, Array, Astra Zeneca, Bayer, Beigene, BMS, Casi, Celgene, Exelixis, Genentech, Halozyme, Incyte, Mabvax, Polaris Puma, QED, Roche, and consulting suppport from Agios, Astra Zeneca, Autem, Bayer, Beigene, Berry Genomics, Bioline, BMS, Celgene, CytomX, Debio, Eisai, Exelixis, Flatiron, Genoscience, Incyte, Ipsen, Jansen, LAM, Lilly, Loxo, Merck, MINA, Pfizer, QED, Redhill, Silenseed, Sillajen, Sobi, Targovax, Therabionics, Twoxar, Yiviva. The authors have no other relevant affiliations or financial involvement with any organization or entity with a financial interest in or financial conflict with the subject matter or materials discussed in the manuscript.

No writing assistance was utilized in the production of this manuscript 


\section{Open access}

This work is licensed under the Attribution-NonCommercial-NoDerivatives 4.0 Unported License. To view a copy of this license, visit http://creativecommons.org/licenses/by-nc-nd/4.0/ 